\title{
SEMIRINGS WITH A COMPLETELY SIMPLE ADDITIVE SEMIGROUP
}

\author{
MIREILLE P. GRILLET
}

(Received 26 February 1973)

Communicated by G. B. Preston

We construct all semirings with a given completely simple additive serricroup $\mathscr{M}(G, I, \wedge, P)$ by means of an associative and distributive multiplication on $G$, and associative multiplications on $I$ and $\wedge$ satisfying certain conditions.

Let $R$ be a semiring such that $(R,+)$ is a Rees matrix semigroup over an (additive) group $G$ with sandwich matrix $P=\left(p_{\lambda, i}\right)$; we select elements $0 \in I$ and $0 \in \wedge$ such that $p_{0, i}=p_{\lambda, 0}=0$ for all $i \in I$ and $\lambda \in \wedge$. Our main result then shows that, given associative multiplications on $I$ and $\wedge$, and a multiplication on $G$, which together with the + , turns $G$ into a semiring with zero satisfying the conditions of Theorem 4 below, then $R$ together with the multiplication defined by:

$$
(i, a, \lambda)(j, b, \mu)=\left(i j,-p_{\lambda \mu, i j}+p_{\lambda \mu, 00}+a b-p_{\lambda \mu, 00}, \lambda \mu\right)
$$

for all $(i, a, \lambda),(j, b, \mu) \in R$, is a semiring. Conversely, every semiring with a completely simple additive semigroup is isomorphic to such a semiring.

Properties of semirings whose additive semigroup is completely simple are studied in Section 1, including some preliminary results on their structure. Our main result is proved in Section 2, while in Section 3, we give interesting particular cases and examples.

Recall that a semiring $R$ is a set with two associative operations,$+ \cdot$ such that $x(y+z)=x y+x z,(x+y) z=x z+y z$ for all $x, y, z \in R$; no other assumption is made. A zero element of $R$ is an element 0 such that $0 x=x 0=0,0+x$ $=x+0=x$ for all $x \in R$.

\section{Preliminary results}

Let $R$ be any semiring whose additive semigroup $(R,+)$ is completely simple. We can represent $(R,+)$ as a Rees $I \times \wedge$ - matrix semigroup $\mathscr{M}(G, I, \wedge, P)$ over an (additive) group $G$ with sandwich matrix $P=\left(p_{\lambda, i}\right)$, and thus write $(R,+)$ as the set of all triples $(i, a, \lambda)$ with $i \in I, \lambda \in \wedge$ and $a \in G$, together with the addition defined by: 


$$
(i, a, \lambda)+(j, b, \mu)=\left(i, a+p_{\lambda, j}+b, \mu\right)
$$

for all $i, j \in I, a, b \in G$ and $\lambda, \mu \in \wedge$. Furthermore we shall select elements $0 \in I$ and $0 \in \wedge$, and assume with no loss in generality that $p_{\lambda, 0}=p_{0, i}=0$ for all $i \in I$ and $\lambda \in \wedge$.

Since the additive Green's relation $\mathscr{L}$ and $\mathscr{R}$ are multiplicative congruences, it is clear that the multiplication of $R$ induces associative multiplications on $I$ and $\wedge$ so that, for all $(i, a, \lambda),(j, b, \mu) \in R$, we have:

$$
(i, a, \lambda)(j, b, \mu)=(i j, m, \lambda \mu)
$$

for some $m=m(i, j, a, b, \lambda, \mu) \in G$.

The additive idempotents of $R$ are the elements $e_{i, \lambda}=\left(i,-p_{\lambda, i}, \lambda\right)$, with $i \in I$, $\lambda \in \wedge$. We denote by $E$ the set of these additive idempotents; trivially, whenever $e_{i, \lambda} \in E$ and $(j, b, \mu) \in R$, we have:

$$
e_{i, \lambda}(j, b, \mu)=e_{i j, \lambda \mu},(j, b, \mu) e_{i, \lambda}=e_{j i, \mu \lambda} .
$$

THEOREM 1. Let $R$ be a semiring such that $(R,+)=\mathscr{M}(G, I, \wedge, P)$. Then, for every $i, j, k \in I$ and $\lambda, \mu, v \in \wedge$, we have:

(1) $p_{\lambda \mu, k j}=p_{\lambda \mu, i j}-p_{v \mu, i j}+p_{v \mu, k j}$

(2) $p_{\mu \lambda, j k}=p_{\mu \lambda, j i}-p_{\mu v, j i}+p_{\mu v, j k}$.

Proof. Let $i, j, k \in I$ and $\lambda, \mu, v \in \wedge$. Then

$$
\begin{aligned}
\left(i j,-p_{v \mu, i j}, v \mu\right) & =e_{i j, v \mu}=\left(i, p_{\lambda, k}, v\right) e_{j, \mu}=((i, 0, \lambda)+(k, 0, v)) e_{j, \mu} \\
& =(i, 0, \lambda) e_{j, \mu}+(k, 0, v) e_{j, \mu}=e_{i j, \lambda \mu}+e_{k j, v \mu} \\
& =\left(i j,-p_{\lambda \mu, i j}, \lambda \mu\right)+\left(k j,-p_{v \mu, k j}, v \mu\right) \\
& =\left(i j,-p_{\lambda \mu, i j}+p_{\lambda \mu, k j}-p_{v \mu, k j}, v \mu\right) .
\end{aligned}
$$

Therefore $-p_{v \mu, i j}=-p_{\lambda \mu, i j}+p_{\lambda_{\mu}, k j}-p_{v \mu, k j}$ and (1) holds. Formula (2) is proved similarly.

Next we study the products in $R$ of the type $(0, a, 0)(0, b, 0)$, where $a, b \in G$. Clearly the multiplication * defined on $G$ by: $(0, a, 0)(0, b, 0)=(00, a * b, 00)$ fails to be distributive with respect to the addition of $G$. However this can be corrected by adding the constant $p_{00,00}$ to $a * b$, and our next result shows that the multiplition of $R$ induces a structure of skew-ring ( $=$ semiring whose additive semigroup is a group) with zero on $G$.

THEOREM 2. Let $R$ be a semiring such that $(R,+)=\mathscr{M}(G, I, \wedge, P)$. Then the multiplication defined on $G$ by:

$$
(0, a, 0)(0, b, 0)=\left(00, a b-p_{00,00}, 00\right)
$$

for all $a, b \in G$ is associative and distributive with respect to the addition of $G$. Furthermore 0 is a multiplicative zero, and for all $i \in I, \lambda \in \wedge, a \in G$ we have: 
(3) $p_{\lambda, i} a=a p_{\lambda, i}=0$.

ProOF. We first check the distributivity. For all $a, b, c \in G$, we have:

$$
\begin{aligned}
\left(00,(a+b) c-p_{00,00}, 00\right) & =(0, a+b, 0)(0, c, 0) \\
& =(0, a, 0)(0, c, 0)+(0, b, 0)(0, c, 0) \\
& =\left(00, a c-p_{00,00}, 00\right)+\left(00, b c-p_{00,00}, 00\right) \\
& =\left(00, a c+b c-p_{00,00}, 00\right) .
\end{aligned}
$$

Thus $(a+b) c=a c+b c$ for all $a, b, c \in G$. Distributivity on the left is checked similarly.

To prove that 0 is a multiplicative zero, let $a \in G$. Then, since $e_{0,0}$ $=(0,0,0) \in E$, we have:

$$
\left(00,-p_{00,00}, 00\right)=e_{00,00}=(0, a, 0)(0,0,0)=\left(00, a 0-p_{00,00}, 00\right) .
$$

Thus $a 0=0$ for all $a \in G$. Similarly $0 a=0$ for all $a \in G$.

Next we show that formula (3) holds. For all $i \in I, \lambda \in \wedge$ and $a \in G$, we have:

Thus we get:

$$
\begin{aligned}
\left(i 0,-p_{\lambda 0, i 0}, \lambda 0\right) & =e_{i 0, \lambda 0}=e_{i, \lambda}(0, a, 0)=\left(e_{i, 0}+\left(0,-p_{\lambda, i}, 0\right)+e_{0, \lambda}\right)(0, a, 0) \\
& =e_{i 0,00}+\left(00,-p_{\lambda, i} a-p_{00,00}, 00\right)+e_{00, \lambda 0} \\
& =\left(i 0,-p_{00, i 0}+p_{00,00}-p_{\lambda, i} a-p_{\lambda 0,00}, \lambda 0\right) .
\end{aligned}
$$

$$
-p_{\lambda 0, i 0}=-p_{00, i 0}+p_{00,00}-p_{\lambda, i} a-p_{\lambda 0,00} \text {. }
$$

This formula holds in particular when $a=0$, and it follows that $p_{\lambda, i} a=p_{\lambda, i} 0=0$. Similarly $a p_{\lambda, i}=0 p_{\lambda, i}=0$. Therefore (3) holds.

Finally to show the associativity, observe that, for all $a, b, c \in G$, we have:

$$
\begin{aligned}
((0, a, 0)(0, b, 0))(0, c, 0) & =\left(00, a b-p_{00,00}, 00\right)(0, c, 0) \\
& =\left(e_{00,0}+\left(0, a b-p_{00,00}, 0\right)+e_{0,00}\right)(0, c, 0) \\
& =e_{000,00}+\left(00,\left(a b-p_{00,00}\right) c-p_{00,00}, 00\right)+e_{00,000} \\
& =\left(000,-p_{00,000}+p_{00,00}+(a b) c-p_{00,00} c-p_{000,00}, 000\right) \\
& =\left(000,-p_{00,000}+p_{00,00}+(a b) c-p_{000,00,000)}\right.
\end{aligned}
$$

by distributivity in $G$ and (3). Similarly we have:

$$
(0, a, 0)((0, b, 0)(0, c, 0))=\left(000,-p_{00,000}+p_{00,00}+a(b c)-p_{000,00}, 000\right)
$$

By associativity in $R$, we therefore have $a(b c)=(a b) c$ for all $a, b, c \in G$, which completes the proof.

It is now clear that, by representing any two elements $(i, a, \lambda)$ and $(j, b, \mu)$ of $R$ as sums 


$$
\begin{aligned}
& (i, a, \lambda)=e_{i, 0}+(0, a, 0)+e_{0, \lambda} \\
& (j, b, \mu)=e_{j, 0}+(0, b, 0)+e_{0 \mu},
\end{aligned}
$$

and using the distributivity laws in $R$ and the relations given above, we can express the product $(i, a, \lambda)(j, b, \mu)$ under various forms in terms of $P$, and products in $I, \wedge$ and $G$. For instance, for all $(i, a, \lambda),(j, b, \mu) \in R$, we have the following:

$$
\begin{aligned}
(i, a, \lambda)(j, b, \mu) & =\left(e_{i, 0}+(0, a, 0)+e_{0, \lambda}\right)(j, b, \mu) \\
& =e_{i, 0}(j, b, \mu)+(0, a, 0)(j, b, \mu)+e_{0, \lambda}(j, b, \mu) \\
& =e_{i j, 0 \mu}+(0, a, 0)\left(e_{j, 0}+(0, b, 0)+e_{0, \mu}\right)+e_{0 j, \lambda \mu} \\
& =e_{i j, 0 \mu}+(0, a, 0) e_{j, 0}+(0, a, 0)(0, b, 0)+(0, a, 0) e_{0, \mu}+e_{0 j, \lambda \mu} \\
& =e_{i j, 0 \mu}+e_{0 j, 00}+\left(00, a b-p_{00,00}, 00\right)+e_{00,0 \mu}+e_{0 j, \lambda \mu} \\
& =\left(i j,-p_{0 \mu, i j}+p_{0 \mu, 0 j}-p_{00,0 j}+p_{00,00}+a b-p_{0 \mu, 00}\right. \\
& \left.\quad+p_{0 \mu, j}-p_{\lambda \mu, 0 j}, \lambda \mu\right) .
\end{aligned}
$$

From (2), we have:

hence

$$
\begin{aligned}
& p_{0 \mu, 00}=p_{0 \mu, 0 j}-p_{00,0 j}+p_{00,00} \\
& p_{00,0 j}=p_{00,00}-p_{0 \mu, 00}+p_{0 \mu, 0 j}
\end{aligned}
$$

(8) $(i, a, \lambda)(j, b, \mu)=\left(i j,-p_{0 \mu, i j}+p_{0 \mu, 00}+a b-p_{00,00}+p_{00,0 j}-p_{\lambda \mu, 0 j}, \lambda \mu\right)$.

A similar transformation of the product $(i, a, \lambda)(j, b, \mu)$ also gives the following expression:

(9) $(i, a, \lambda)(j, b, \mu)=\left(i j,-p_{\lambda 0, i j}+p_{\lambda 0,00}+a b-p_{00,00}+p_{00, i 0}-p_{\lambda \mu, i 0}, \lambda \mu\right)$.

The final result of this section is obtained by comparing these two expressions $(i, a, \lambda)(j, b, \mu)$ successively in the two cases when $j=0, \lambda=0$ and $i=0, \mu=0$.

Proposition 3. Let $R$ be a semiring such that $(R,+)=\mathscr{M}(G, I, \wedge, P)$. Then we have

$$
a b=k+a b-k
$$

where $k=-p_{00,00}+p_{00, i 0}-p_{0 \mu, i 0}+p_{0 \mu, 00}$

$$
a b=k^{\prime}+a b-k^{\prime},
$$

where $k^{\prime}=-p_{00,00}+p_{00,0 j}-p_{\lambda 0,0 j}+p_{\lambda 0,00}$.

\section{The main result}

THEOREM 4. Let $R=\mathscr{M}(G, I, \wedge, P)$ be an additive Rees $I \times \wedge$-matrix semigroup over the (additive) group $G$, and let $0 \in I, 0 \in \wedge$ be such that $p_{\lambda} 0$ 
$=p_{0, i}=0$ for all $i \in I, \lambda \in \wedge$. Given associative multiplications on $I$ and $\wedge$, and a structure of skew-ring with zero on $G$ satisfying the following conditions:

(1) $p_{\lambda \mu, k j}=p_{\lambda \mu, i j}-p_{v \mu, i j}+p_{v \mu, k j}$

(2) $p_{\mu \lambda, j k}=p_{\mu \lambda, j i}-p_{\mu v, j i}+p_{\mu v, j k}$

(3) $p_{\lambda, i} a=a p_{\lambda, i}=0$

(4) $a b=k+a b-k$,

where $k=-p_{00,00}+p_{00, i 0}-p_{0 \mu, i 0}+p_{0 \mu, 00}$,

(5) $a b=k^{\prime}+a b-k^{\prime}$, where $k^{\prime}=-p_{00,00}+p_{000 j}-p_{\lambda 0,0 j}+p_{\lambda 0,00}$, for all $i, j, k \in I, \lambda, \mu, v \in \wedge$ and $a, b \in G$; then $R$ together with the multiplication defined by:

(6) $(i, a, \lambda)(j, b, \mu)=\left(i j,-p_{\lambda \mu, i j}+p_{\lambda \mu}, 00+a b-p_{\lambda \mu}, 00, \lambda \mu\right)$ for all $(i, a, \lambda),(j, b, \mu) \in R$, is a semiring.

Conversely every semiring with a completely simple additive semigroup is isomorphic to such a semiring.

Proof. Let $R$ be as in the statement of the first part of the theorem, and consider on $R$ the multiplication defined by formula (6). We first check that this multiplication is distributive with respect to the addition. For all $x=(i, a, \lambda)$, $y=(j, b, \mu)$ and $z=(k, c, v)$ belonging to $R$, we have:

while

$$
\begin{aligned}
x y+x z= & \left(i j,-p_{\lambda_{\mu}, i j}+p_{\lambda_{\mu}, 00}+a b-p_{\lambda \mu, 00}, \lambda \mu\right) \\
& +\left(i k,-p_{\lambda v, i k}+p_{\lambda_{v}, 00}+a c-p_{\lambda v, 00}, \lambda v\right) \\
= & \left(i j,-p_{\lambda \mu, i j}+p_{\lambda \mu, 00}+a b-p_{\lambda_{\mu}, 00}+p_{\lambda_{\mu}, i k}-p_{\lambda v, i k}\right. \\
& \left.\quad+p_{\lambda v, 00}+a c-p_{\lambda v, 00}, \lambda v\right),
\end{aligned}
$$

$$
\begin{aligned}
x(y+z) & =(i, a, \lambda)\left(j, b+p_{\mu, k}+c, v\right) \\
& =\left(i j,-p_{\lambda v, i j}+p_{\lambda v, 00}+a\left(b+p_{\mu, k}+c\right)-p_{\lambda v, 00}, \lambda v\right) \\
& =\left(i j,-p_{\lambda,, i j}+p_{\lambda v, 00}+a b+a c-p_{\lambda v, 00}, \lambda v\right),
\end{aligned}
$$

since by (3) we have $a p_{\mu, k}=0$.

To prove that $x y+x z=x(y+z)$, it is then clearly enough to show that, if

$$
\begin{aligned}
& m=-p_{\lambda v, 00}+p_{\lambda v, i j}-p_{\lambda \mu, i j}+p_{\lambda \mu, 00} \\
& m^{\prime}=-p_{\lambda v, 00}+p_{\lambda v, i k}-p_{\lambda \mu, i k}+p_{\lambda \mu, 00},
\end{aligned}
$$

then $m+a b=a b+m^{\prime}$. Consider first the expression of $m$. By (2) we have

whence

$$
p_{\lambda v, i j}-p_{\lambda \mu, i j}=p_{\lambda v, i 0}-p_{\lambda_{\mu}, i 0}
$$

$$
m=-p_{\lambda v, 00}+p_{\lambda v, i 0}-p_{\lambda_{\mu, i 0}}+p_{\lambda_{\mu}, 00}
$$

Since by (1) we also have: 
we get:

$$
\begin{aligned}
-p_{\lambda v, 00}+p_{\lambda v, i 0} & =-p_{0 v, 00}+p_{0 v, i 0} \\
-p_{\lambda \mu, i 0}+p_{\lambda \mu, 00} & =-p_{0 \mu, i 0}+p_{0 \mu, 00}
\end{aligned}
$$

$$
m=-p_{0 v, 00}+p_{0 v, i 0}-p_{0 \mu, i 0}+p_{0 \mu, 00}
$$

Since this expression does not depend on $j$, we get at once:

$$
m^{\prime}=m \text {. }
$$

Then, using twice formula (4), we have:

$$
\begin{aligned}
m+a b= & -p_{0 v, 00}+p_{0 v, i 0}-p_{0 \mu, i 0}+p_{0 \mu, 00}+a b \\
= & \left(-p_{0 v, 00}+p_{0 v, i 0}-p_{00, i 0}+p_{00,00}\right)+\left(-p_{00,00}+p_{00, i 0}\right. \\
& \left.\quad-p_{0 \mu, i 0}+p_{0 \mu, 00}\right)+a b \\
= & \left(-p_{0 v, 00}+p_{0 v, i 0}-p_{00, i 0}+p_{00,00}\right)+a b+\left(-p_{00,00}+p_{00, i 0}\right. \\
& \left.\quad-p_{0 \mu, i 0}+p_{0 \mu, 00}\right) \\
= & a b-p_{0 v, 00}+p_{0 v, i 0}-p_{00, i 0}+p_{00,00}-p_{00,00}+p_{00, i 0} \\
& \quad-p_{0 \mu, i 0}+p_{0 \mu, 00} \\
= & a b-p_{0 v, 00}+p_{0 v, i 0}-p_{0 \mu, i 0}+p_{0 \mu, 00}=a b+m^{\prime} .
\end{aligned}
$$

Thus distributivity on the left holds. That distributivity on right holds too is proved similarly using formula (5) instead of formula (4).

Associativity of the multiplication of $R$ follows then from Proposition 1.1 of Grillet (197a), since it is easily seen that the subset $E \cup H_{0,0}$ of $R$ is a generating subset of $(R,+)$ which also generates a multiplicative subsemigroup. This completes the proof of the direct part of the theorem.

For the converse, assume that $R$ is a semiring such that $(R,+)$ $=\mathscr{M}(G, I, \wedge, P)$. We already have proved in section 1 that the conditions stated in the direct part hold, and also that, for all $x=(i, a, \lambda), y=(j, b, \mu) \in R$, we have, by (8):

$x y=(i, a, \lambda)(j, b, \mu)=\left(i j,-p_{0 \mu, i j}+p_{0 \mu, 00}+a b-p_{00,00}+p_{00,0 j}-p_{\lambda \mu, 0 j}, \lambda \mu\right)$.

By (2), we have: $-p_{\lambda_{\mu}, 0 j}=-p_{\lambda, 0,0 j}+p_{\lambda 0,00}-p_{\lambda \mu, 00}$, whence

$x y=\left(i j,-p_{0 \mu, i j}+p_{0 \mu, 00}+a b-p_{00,00}+p_{00,0 j}-p_{\lambda 0,0 j}+p_{\lambda 0,00}-p_{\lambda \mu, 00}, \lambda \mu\right)$.

From (5), it then follows that

$x y=\left(i j,-p_{0 \mu, i j}+p_{0 \mu, 00}-p_{00,00}+p_{00,0 j}-p_{\lambda 0,0 j}+p_{\lambda, 0,00}+a b-p_{\lambda_{\mu}, 00}, \lambda \mu\right)$.

Since by (2) $p_{0 \mu, 00}-p_{00,00}+p_{00,0 j}=p_{0 \mu, 0 j}$, we have:

$x y=\left(i j,-p_{0 \mu, l j}+p_{0 \mu, 0 j}-p_{\lambda 0,0 j}+p_{\lambda 0,00}+a b-p_{\lambda \mu, 00, \lambda \mu)}\right.$ 


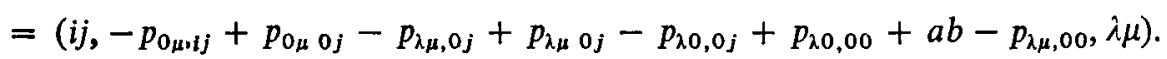

By (1) $-p_{0 \mu, i j}+p_{0 \mu, 0 j}-p_{\lambda \mu, 0 j}=-p_{\lambda \mu, i j}$, and by (2) $p_{\lambda \mu, 0 j}-p_{\lambda 0_{0,0 j}}+p_{\lambda 0,00}$ $=p_{\lambda \mu, 00}$, so that

$$
x y=\left(i j,-p_{\lambda \mu, i j}+p_{\lambda \mu, 00}+a b-p_{\lambda \mu, 00}, \lambda \mu\right) .
$$

Therefore (6) holds which completes the proof.

We give now alternate descriptions of the multiplication on $R$ given in Theorem 4, which have interest of their own.

Proposition 5. Given the data in Theorem 4, the following formulae

(6) $x y=\left(i j,-p_{\lambda \mu, i j}+p_{\lambda \mu, 00}+a b-p_{\lambda \mu, 00}, \lambda \mu\right)$

(7) $x y=\left(i j,-p_{00, i j}+p_{00,00}+a b-p_{00,00}+p_{00, i j}-p_{\lambda \mu, i j}, \lambda \mu\right)$

(8) $x y=\left(i j,-p_{0 \mu, i j}+p_{0 \mu, 00}+a b-p_{00,00}+p_{00,0 j}-p_{\lambda \mu, 0 j}, \lambda \mu\right)$

(9) $x y=\left(i j,-p_{\lambda,,_{i j}}+p_{\lambda, 0,00}+a b-p_{00,00}+p_{00, i 0}-p_{\lambda \mu, i 0}, \lambda \mu\right)$

for all $x=(i, a, \lambda), y=(j, b, \mu) \in R$, define the same multiplication on $R$.

Proof. By Theorem 4, we know that formula (6) defines a structure of semiring on $R$. We then proved in section 1 that (8) and (9) hold, and thus clearly define the same multiplication on $R$ as (6). To complete the proof, we have left to show that formula (7) holds in $R$. Let $x=(i, a, \lambda), y=(j, b, \mu) \in R$. Starting with (8), since by (2) $-p_{0 \mu, i j}=-p_{00, i j}+p_{00, i 0}-p_{0 \mu, i 0}$ we have:

$$
\begin{gathered}
x y=\left(i j,-p_{00, i j}+p_{00, i 0}-p_{0 \mu, i 0}+p_{0 \mu, 00}+a b-p_{00,00}\right. \\
+p_{00,0 j}-p_{\left.\lambda_{\mu, 0 j}, \lambda \mu\right) .}
\end{gathered}
$$

By (4), it follows that

$$
\begin{aligned}
x y=\left(i j,-p_{00, i j}\right. & +p_{00,00}+a b-p_{00,00}+p_{00, i 0}-p_{0 \mu, i 0} \\
& \left.+p_{0 \mu, 00}-p_{00,00}+p_{00,0 j}-p_{\lambda \mu, 0 j}, \lambda \mu\right) .
\end{aligned}
$$

Again by (2), we have: $p_{0 \mu, 00}-p_{00,00}+p_{00,0 j}=p_{0 \mu, 0 j}$, so that

$$
\begin{aligned}
x y=\left(i j,-p_{00, i j}\right. & +p_{00,00}+a b-p_{00,00}+p_{00, i 0}-p_{0 \mu, i 0} \\
& \left.+p_{0 \mu, 0 j}-p_{\lambda \mu, 0 j}, \lambda \mu\right) \\
=\left(i j,-p_{00, i j}\right. & +p_{00,00}+a b-p_{00,00}+p_{00, i 0}-p_{0 \mu, i 0} \\
& \left.+p_{0 \mu, i j}-p_{0 \mu, i j}+p_{0 \mu, 0 j}-p_{\lambda \mu, 0 j}, \lambda \mu\right) .
\end{aligned}
$$

Now by (2) $p_{00, i 0}-p_{0 \mu, i 0}+p_{0 \mu, i j}=p_{00, i j}$, and by (1) $-p_{0 \mu, i j}+p_{0 \mu, 0 j}-p_{\lambda \mu, 0 j}$ $=-p_{\lambda \mu, i j}$, whence

$$
x y=\left(i j,-p_{00, i j}+p_{00,00}+a b-p_{00,00}+p_{00, i j}-p_{\lambda \lambda \mu, i j}, \lambda \mu\right),
$$

which proves that (7) holds.

Corresponding to the situation when $(0, a, 0)(0, b, 0)=\left(00,-p_{00,00}+a b, 00\right)$ 
instead of $(0, a, 0)(0, b, 0)=\left(00, a b-p_{00,00}, 00\right)$, we clearly have a result dual to Theorem 4; it can be stated by replacing in Theorem 4 formulae (4), (5) and (6) respectively by

$$
a b=h+a b-h
$$

where $h=p_{00, i 0}-p_{0 \mu, i 0}+p_{0 \mu, 00}-p_{00,00}$;

$$
a b=h^{\prime}+a b-h^{\prime}
$$

where $h^{\prime}=p_{00,0 j}-p_{\lambda 0,0 j}+p_{00,0 j}-p_{00,00}$;

$$
(i, a, \lambda)(j, b, \mu)=\left(i j,-p_{00, i j}+a b+p_{00, i j}-p_{\lambda \mu-i j}, \lambda \mu\right) .
$$

We shall give below an example showing that in general $p_{00,00}$ does not commutes with products $a b$ of elements $a, b$ of $G$. Thus even if all conditions involved in both statements are satisfied, these theorems usually give different semiring structures on $R$. We note that, if we assume that $p_{00,00}$ commutes with all products of elements of $R$, then, trivially, conditions $\left(4^{\prime}\right)$ and $\left(5^{\prime}\right)$ are respectively equivalent to (4) and (5), and in view of Proposition 5, the two multiplications defined by formulae (6) and $\left(6^{\prime}\right)$ are the same.

A last remark we can make about Theorem 4 is that, though for practical reasons we broke our conditions as much as possible, it is possible to condense conditions (4) and (5) into only one condition. For instance, it is easy to see that, assuming that (2) and (3) hold, conditions (4) and (5) together are equivalent to the following:

$$
a b=m+a b-m
$$

where $m=-p_{00,00}+p_{00 i j}-p_{\lambda \mu, i j}+p_{\lambda \mu, 00}$.

\section{Examples and applications}

We start this section by giving two examples showing that there is little hope of simplifying the conditions of theorem 4 . First we note that the formula

$$
p_{\lambda \mu, i j}=p_{\lambda \mu, k l}-p_{v \rho, k l}+p_{v \rho, i j}
$$

does not need to hold in general as shown by the following:

EXAmple 6. Let $R=\mathscr{M}(G, I, \wedge, P)$, where

$G=\{0, a\}$ together with the addition: $0+0=a+a=0,0+a=a+0$ $=a$, and the trivial multiplication;

$I=\{0,1\}$ with multiplication: $00=01=0,10=11=1$;

$\wedge=\{0,1\}$ with multiplication: $00=10=0,01=11=1$;

$$
p_{0,0}=p_{0,1}=p_{1,0}=0, \quad p_{1,1}=a .
$$


Observe that the conditions of Theorem 4 are trivially satisfied so that formula 6 defines a structure of semiring on $R$. However we have: $a=p_{11,11}$ $\neq p_{11,00}-p_{00,00}+p_{00,11}=0$.

Next example shows that in general $p_{00,00}$ does not commute with products of elements of $G$.

ExAmple 7. Let $R=\mathscr{M}(G, I, \wedge, P)$, where $G$ is the skew-ring given by the following tables:

\begin{tabular}{l|llllll}
+ & 0 & $a$ & $b$ & $c$ & $d$ & $e$ \\
\hline 0 & 0 & $a$ & $b$ & $c$ & $d$ & $e$ \\
$a$ & $a$ & $b$ & 0 & $e$ & $c$ & $d$ \\
$b$ & $b$ & 0 & $a$ & $d$ & $e$ & $c$ \\
$c$ & $c$ & $d$ & $e$ & 0 & $a$ & $b$ \\
$d$ & $d$ & $e$ & $c$ & $b$ & 0 & $a$ \\
$e$ & $e$ & $c$ & $d$ & $a$ & $b$ & 0
\end{tabular}

\begin{tabular}{l|llllll}
$\cdot$ & 0 & $a$ & $b$ & $c$ & $d$ & $e$ \\
\hline 0 & 0 & 0 & 0 & 0 & 0 & 0 \\
$a$ & 0 & 0 & 0 & 0 & 0 & 0 \\
$b$ & 0 & 0 & 0 & 0 & 0 & 0 \\
$c$ & 0 & 0 & 0 & $c$ & $c$ & $c$ \\
$d$ & 0 & 0 & 0 & $c$ & $c$ & $c$ \\
$e$ & 0 & 0 & 0 & $c$ & $c$ & $c$
\end{tabular}

$I=\{0,1\}, \quad \wedge=\{0,1\} \quad$ with $I^{2}=\{1\} ; \quad \wedge^{2}=\{1\} ; \quad p_{0,0}=p_{1,0}=p_{0,1}=0$, $p_{1,1}=a$. Then formula 6 trivially defines a structure of semiring on $R$. Observe that

$$
e=a+c=p_{00,00}+c^{2} \neq c^{2}+p_{00,00}=c+a=d .
$$

An important case when the conditions of theorem 4 are trivially satisfied is when $R$ is a rectangular group, in which case we may assume that $P=0$.

COROLlaRY 8. Let $R=\mathscr{M}(G, I, \wedge, 0)$ be a rectangular group. Then given ussociative multiplications on $I$ and $\wedge$, and a structure of skew-ring on $G, R$ tojether with the multiplication defined by:

$$
(i, a, \lambda)(j, b, \mu)=(i j, a b, \lambda \mu)
$$

or all $(i, a, \lambda),(j, b, \mu) \in R$, is a semiring. Conversely every semiring whose addiive semigroup is a rectangular group is isomorphic to such a semiring.

Note that, if $(G, \cdot)$ has no divisor of zero, then formula (3) trivially implies hat $P=0$. Another example where $(R,+)$ must be a rectangular group is given $y$ the following.

Proposition 9. Let $R$ be a semiring whose additive semigroup is a comletely simple semigroup, and such that $R=R^{2}$. If there exist a $\mathscr{L}$-class and a 
$\mathscr{R}$-class of $R$ which are left (right) ideals of $(R \cdot)$, then $(R,+)$ is a rectangular group.

Proof. Let $(R,+)=\mathscr{M}(G, I, \wedge, P)$, and assume for instance that $R$ contains a $\mathscr{L}$-class $L$ and a $\mathscr{R}$-class $S$ which are left ideals of $(R, \cdot)$. We may assume that $L$ and $S$ contain $(0,0,0)$, so that $L=\{(i, a, 0) ; a \in G, i \in I\}$ and $S=\{(0, a, \lambda) ; a \in G, \lambda \in \wedge\}$. Then clearly $L R \subseteq L$ and $S R \subseteq S$ imply that $0 i=0$, $0 \lambda=0$ for all $i \in I$ and $\lambda \in \wedge$. By (1) we have:

$$
p_{\lambda \mu, i j}=p_{\lambda \mu, 0 j}-p_{0 \mu, 0 j}+p_{0 \mu, i j}=p_{\lambda \mu, 0}-p_{0,0}+p_{0, i j}=0
$$

for all $i, j \in I$ and $\lambda, \mu \in \wedge$. Since $R=R^{2}$, we have $I=I^{2}$ and $\wedge=\wedge^{2}$, and it follows that $p_{\lambda, i}=0$ for all $i \in I$ and $\lambda \in \wedge$, which completes the proof.

The assumptions of the theorem hold in particular in case all $\mathscr{L}$-classes and all $\mathscr{R}$-classes of $R$ are left (right) ideals of $(R, \cdot)$, i.e. when $I$ and $\wedge$ are left (right) zero-semigroups. An interesting particular case of Theorem 4 is also obtained in case $I$ is a left (right) zero-semigroup and $\wedge$ is a right (left) zero-semigroup. For instance, we have:

CoROllary 10. Let $R=\mathscr{M}(G, I, \wedge, P)$ be an additive $I \times \wedge$-matrix semigroup over the (additive) group $G, 0 \in I$ and $0 \in \wedge$ be such that $p_{\lambda, 0}=p_{0 . i}=0$ for all $i \in I, \lambda \in \wedge$. Given a structure of skew-ring with zero on $G$ satisfying the conditions

i) $p_{\lambda, i} a=a p_{\lambda, i}=0$

ii) $a+p_{\lambda, i}=p_{\lambda, i}+a$

for all $a \in G^{2}, i \in I$ and $\lambda \in \wedge$. Then $R$ together with the multiplication defined by:

$$
(i, a, \lambda)(j, b, \mu)=\left(i,-p_{\lambda, j}+a b, \mu\right)
$$

for all $(i, a, \lambda),(j, b, \mu) R$ is a semiring such that all $\mathscr{L}$-classes ( $\mathscr{R}$-classes) of $R$ are left (right) ideals of $(R, \cdot)$. Conversely every semiring with a completely simple additive semigroup whose $\mathscr{L}$-classes ( $\mathscr{R}$-classes) are left (right) ideals of $(R, \cdot)$ is isomorphic to such a semiring.

Proof. Trivially under the further assumption that all $\mathscr{L}$-classes ( $\mathscr{R}$-classes) of $R$ are left (right) ideals of $(R, \cdot)$, the conditions of Theorem 4 reduce to the two above conditions. The result then follows

Our last result restates Theorem 4 in case $G$ is an abelian group. Then conditions (4) and (5) are trivially satisfied, and formula (6) takes a simpler form. We then have the following:

Corollary 11. Let $R=\mathscr{M}(G, I, \wedge, P)$ be an additive Rees $I \times \wedge$-matrix semigroup over an (additive) abelian group $G, 0 \in I$ and $0 \in \wedge$ be such that $p_{\lambda, 0}=p_{0, i}=0$ for all $i \in I$ and $\lambda \in \wedge$. Given associative multiplications on $I$ and $\wedge$, and a structure of ring on $G$ satisfying the following conditions: 


$$
\begin{aligned}
p_{\lambda \mu, k j} & =p_{\lambda \mu, i j}-p_{v \mu, i j}+p_{v \mu, k j} \\
p_{\mu \lambda, j k} & =p_{\mu \lambda, j i}-p_{\mu v j i}+p_{\mu v, j k} \\
p_{\lambda, i} a & =a p_{\lambda, i}=0
\end{aligned}
$$

for all $i, j \in I, \lambda, \mu \in \wedge$ and $a \in G$, then $R$ together with the multiplication defined by:

$$
(i, a, \lambda)(j, b, \mu)=\left(i j,-p_{\lambda \mu, i j}+a b, \lambda \mu\right)
$$

for all $(i, a, \lambda),(j, b, \mu) \in R$, is a semiring. Conversely every semiring whose additive semigroup is a Rees $I \times \wedge$-matrix semigroup over an abelian group is isomorphic to such a semiring.

\section{References}

A. H. Clifford and G. B. Preston (1962), The algebraic theory of semigroups, Vol. 1, Math. Surveys, No. 7, (Amer. Math. Soc., Providence, R. I., 1962.)

M. P. Grillet (1972), 'Embedding problems in semiring theory', J. Austral. Math. Soc. 22, 168-181.

c/o Pierre A. Grillet

Tulane University

New Orleans, La 70118

U.S.A. 